\title{
Evaluación del efecto de las politicas educativas sobre la desigualdad del ingreso en las regiones de Junín, Pasco y Huánucor
}

\section{Evaluation of the effect of education expenditure policies on income inequality in the Junin, Pasco and Huanuco regions}

\author{
Ricardo N. Villamonte Blas ${ }^{2}$ \\ Universidad Nacional Mayor de San Marcos \\ rvillamonteb@unmsm.edu.pe \\ Tomas J. Delgado Chumbile ${ }^{3}$ \\ Universidad Nacional Mayor de San Marcos \\ tomas.delgado@unmsm.edu.pe
}

Recibido: 29/08/2020 - Aceptado: 23/04/2021 - Publicado: 06/12/2021

\begin{abstract}
RESUMEN
El Perú ha tenido un crecimiento económico aceptable en relación a otros países de Latinoamérica en los últimos 10 años. Aun cuando la desigualdad de los ingresos de las personas se ha reducido en el periodo 2007-2017, interesa analizar cuál ha sido el efecto de las políticas educativas en la desigualdad de los ingresos en las regiones de: Pasco, Junín y Huánuco, regiones clasificadas entre las de menores ingresos personales en el Perú.

En los últimos 10 años el gobierno ha ido perfeccionando los mecanismos de acceso a la educación pública y mejorando sus políticas públicas brindando un mayor presupuesto con el fin de lograr un incremento de accesibilidad educativa.

El presente estudio tiene por objetivo realizar un análisis del efecto en la desigualdad de los ingresos medido por el coeficiente de Gini, de un conjunto de variables
\end{abstract}

(C) Los autores. Este artículo es publicado por Pensamiento Crítico de la Facultad de Ciencias Económicas, Universidad Nacional Mayor de San Marcos. Este es un artículo de acceso abierto, distribuido bajo los términos de la licencia Creative Commons Atribucion - No Comercia_Compartir Igual 4.0 Internacional. (http://creativecommons.org/licenses/by-nc-sa/4.0/) que permite el uso no comercial, distribución y reproducción en cualquier medio, siempre que la obra original sea debidamente citada. 
explicativas representativas de las políticas educativas a través de un modelo econométrico de panel data propuesto, luego de una revisión de literatura especializada en la materia y de las principales políticas educativas en el periodo 2007- 2017. La hipótesis de trabajo afirma que las políticas educativas tuvieron un impacto bajo en la reducción de las desigualdades de los ingresos, la misma que se logró comprobar a través de esta investigación.

Palabras claves: políticas educativas; desigualdad de los ingresos; coeficiente de gini; salarios.

JEL: I 21, I 31

\section{ABSTRACT}

Perú has had an acceptable economic growth in relation to other Latin American countries in the last 10 years. Even though people's income inequality has been reduced in the 2007-2017 period, it is interesting to analyze the effect of educational policies on income inequality in the regions of: Pasco, Junín and Huánuco, classified regions among those with the lowest personal income in Perú.

In the last 10 years, the government has been perfecting the mechanisms for access to public education and improving its public policies, providing a larger budget in order to achieve an increase in educational accessibility.

The objective of this study is to carry out an analysis of the effect on income inequality, measured by the Gini coefficient, of a set of representative explanatory variables of educational policies through a proposed panel data econometric model, after a review of specialized literature on the subject and of the main educational policies in the period 2007-2017. The working hypothesis affirms that educational policies had a low impact on reducing income inequalities, the same that was verified through of this investigation.

Keywords: educational policies; income inequality; Gini coefficient; wages. 


\section{Introducción}

El perfeccionamiento en la aplicación de las políticas gubernamentales a futuro requiere del conocimiento y difusión del impacto de las políticas educativas en la desigualdad de los ingresos. Para ello, este artículo considera una breve referencia de la evolución de las variables relevantes para del estudio, el enfoque de los principales organismos internacionales: UNESCO y OCDE, luego se presenta el enfoque de los principales autores en la materia tales como: Becker, Mincer, Beyer y Sen. Finalmente, se presentan la revisión de las apreciaciones y conclusiones de los estudios vinculados a nuestro tema de investigación. Lo mencionado permitió luego formular un modelo econométrico en función de la disponibilidad de datos y arribar a los resultados, discusiones y conclusiones de la presente investigación.

El Perú ha tenido un crecimiento económico destacable a nivel de Latinoamérica en los últimos 10 años, ubicándose entre los 4 primeros, pero los ingresos de las personas siguen siendo muy desiguales.

Los niveles de acceso a la educación pública se han ido incrementando en la medida que las diferentes administraciones de gobierno han mostrado cambios en sus políticas educativas públicas y brindado un mayor presupuesto al sector educación en los últimos 10 años.

En el caso peruano hay cuatro áreas fundamentales para entender el estado de la educación: el acceso y la cobertura educativa, la calidad y el desempeño, la pertinencia de la educación y, la inversión en educación.

La figura $\mathrm{N}^{\circ} 1$ permite apreciar según el coeficiente de gini (medida económica para calcular la desigualdad de ingresos entre los ciudadanos de un territorio) que en el periodo de estudio 2007-2017 para los departamentos de Huánuco, Junín, Pasco se dio una reducción en la desigualdad de los ingresos siendo los coeficientes para los años 2007 y 2017 los siguientes: 0.508 ; $0.444 ; 0.466$ para el año 2007 y $0.449 ; 0.398 ; 0.3821$ para el año 2017 respectivamente. Obsérvese en la figura que, si bien hay una mejora en promedio en los niveles indicados, estos no han sido sostenidos a lo largo del periodo.

Para los mismos años indicados, la mayor disminución de la desigualdad se presentó en la costa urbana (de 0,43 a 0,36), seguido de Lima Metropolitana (de 0,46 a 0,40 ), la selva urbana (de 0,46 a 0,41 ) y la sierra 
urbana (de 0,45 a 0,40). Según Ñopo (2018) el Gini es un indicador que difícilmente se mueve, pues para ello sería necesario cambiar la distribución de ingresos de todo el país. El presente estudio pretende analizar parcialmente el efecto de las políticas educativas en dicho coeficiente.

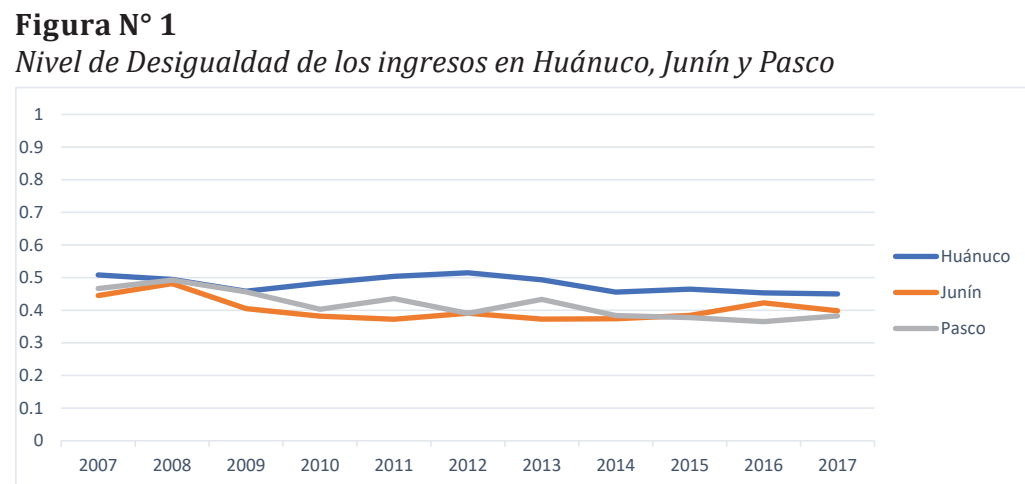

Unidad de Medida: coeficiente de gini Fuente: INEI-ENAHO

La figura $\mathrm{N}^{\circ} 2$ nos muestra según el coeficiente del logaritmo del salario por hora (permite apreciar el análisis marginal del aumento de la variable) para los departamentos en estudio: Huánuco, Junín, Pasco que se ha dado una mejora de los salarios por hora, siendo los coeficientes obtenidos, para el año 2007 los siguientes: -0.193 ; $-0.024 ;-0.052$ y para el año 2017: 0.112; 0.197; 0.164 respectivamente.

Figura $\mathbf{N}^{\circ} \mathbf{2}$

Salario por hora

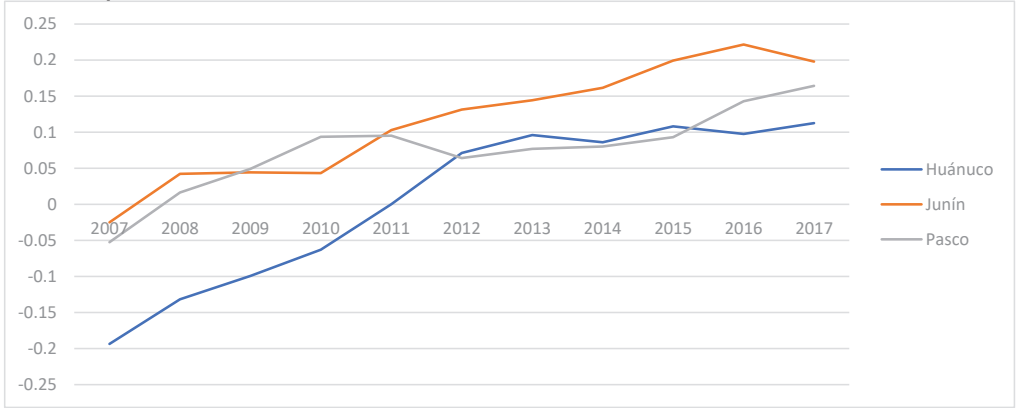

Unidad de medida: logaritmo del salario por hora.

Fuente: INEI 
La figura $\mathrm{N}^{\circ} 3$ permite apreciar la tasa de alumnos que concluyeron el nivel de educación primaria en el periodo de estudio 2007-2017, para los departamentos en estudio: Huánuco, Junín, Pasco se ha dado una mejora en ello, siendo los porcentajes obtenidos, para los años 2007 y 2017: 69.6; 85.9; 82.3 para el año 2007 subieron a 77.7; 91.1; 90.7 para el año 2017 respectivamente.

\section{Figura $\mathbf{N}^{\circ} \mathbf{3}$}

Tasa de alumnos que concluyeron el nivel de educación primaria.

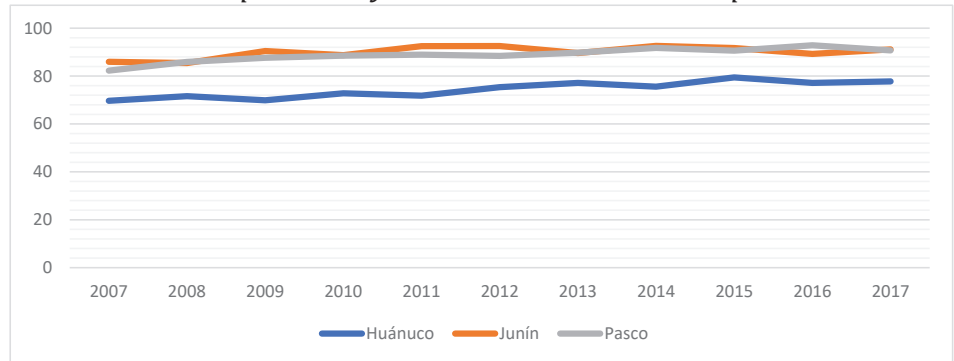

Unidad de medida: porcentaje

Fuente: ESCALE

La figura $\mathrm{N}^{\circ} 4$ permite apreciar la tasa de alumnos que concluyeron el nivel de educación superior en el periodo de estudio 2007-2017 para los departamentos en estudio: Huánuco, Junín, Pasco se ha dado una importante mejora en ello, siendo los porcentajes obtenidos: $13.4 ; 24.4 ; 23.8$ para el año 2007, subieron a 75.9; 73.1; 75.6 para el año 2017 respectivamente.

\section{Figura $\mathbf{N}^{\circ} \mathbf{4}$}

Tasa de alumnos que concluyeron el nivel de educación superior

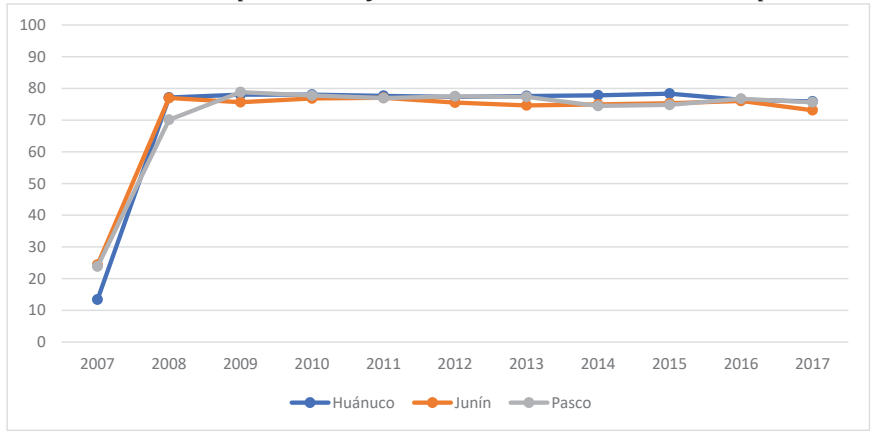

Unidad de medida: porcentaje

Fuente: ESCALE 
La figura $\mathrm{N}^{\circ} 5$ permite apreciar la tasa de analfabetismo en el periodo de estudio 2007-2017 para los departamentos en estudio: Huánuco, Junín, Pasco se ha dado una importante mejora en ello, siendo los porcentajes obtenidos: $17.8 ; 10.9 ; 11.2$ para el año 2007 , subieron a $13.1 ; 5.4 ; 7.3$ para el año 2017 respectivamente.

\section{Figura $\mathbf{N}^{\circ} \mathbf{5}$ \\ Tasa Analfabetismo}

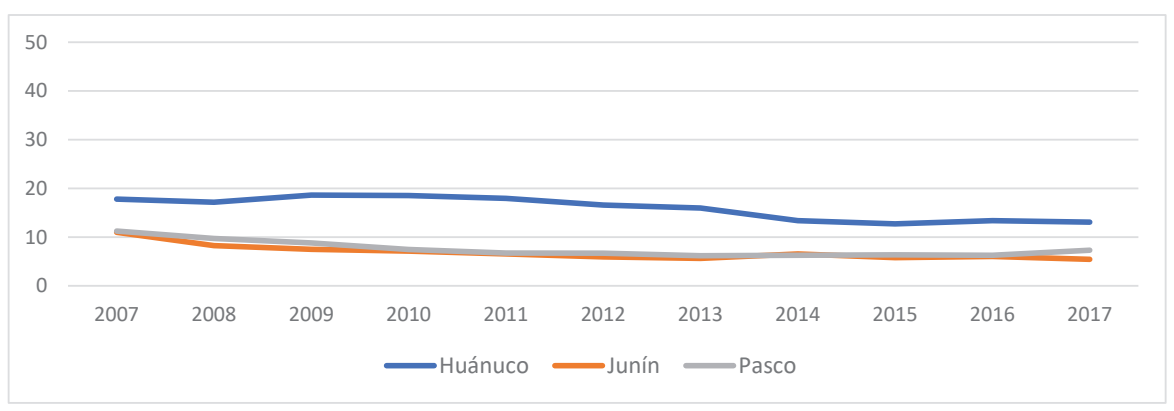

Unidad de medida: Población de 15 años a más que no sabe leer ni escribir. Fuente: INEI

Obsérvese en la figura № 6 la evolución del nivel de la población económicamente activa en el periodo de estudio 2007-2017 para los departamentos en estudio: Huánuco, Junín, Pasco fue de: 76.3, 74.14, 73.15 para el año 2007 paso a ser: 75.8, 73.12 y 75.6 para el año 2017 respectivamente. El comportamiento es irregular para las 3 regiones.

\section{Figura № 6}

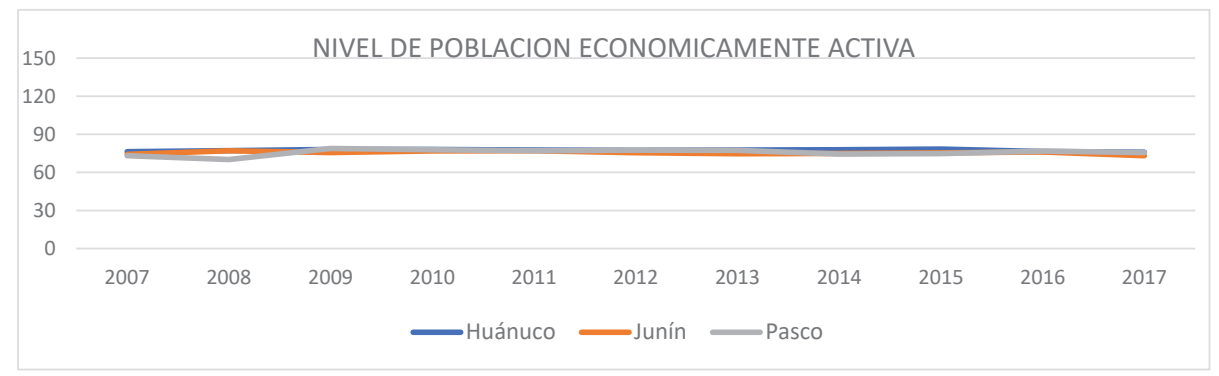

Unidad de medida: PEA ocupa

Fuente INEA 


\section{Marco Teórico}

\subsection{Revisión de la literatura}

La UNESCO (2015), señala que para mejorar el desarrollo mundial se debe velar por la educación en general a fin de que todas las personas tengan la oportunidad de adquirir conocimientos con los que puedan contribuir al desarrollo sostenible de sus países. Dos de sus niveles son los correspondientes son el nivel de educación primaria y superior que se consideran en el presente artículo. Este organismo internacional reconoce la creciente disparidad entre la educación rural y urbana, que las desigualdades internas son muy agudas en prácticamente todos los países de las regiones pobres, siendo la clase social, la condición indígena y la zona de residencia las variables principales que condicionan dichas inequidades. La UNESCO utiliza el inventario de los marcos nacionales de cualificaciones (son los resultados de las evaluaciones del aprendizaje para reformar y ampliar la oferta educativa y la provisión de capacitación) con el objetivo de contribuir a la política y práctica educativa, como parte de su compromiso de promover el aprendizaje a lo largo de toda la vida. Este estudio presenta un análisis estadístico histórico de las medidas tomadas por diferentes gobiernos y concluye que los países enfrentan el desafío de modernizar los marcos de cualificaciones, incorporando no solo mediciones de conocimiento abstracto y complementarlas sino también en lo referido con la acreditación de habilidades, tanto generales como específicas de distintos oficios.

La OCDE (2015), hace referencia a la necesidad de estudiar los principales desafíos del mundo, analizando los avances y políticas implementadas recientemente, y planteando orientaciones de política para el futuro. Desarrollar modelos económicos basados en una mayor diversificación productiva y en fuentes de crecimiento que permitan aportar mayor valor agregado e integrarse de manera más activa en la economía global.

Para la elaboración del modelo econométrico se revisaron autores e investigadores cuyos estudios ayuden, en función a la información estadística disponible, a su construcción cuantitativa e interpretación. Los cuales se resumen a continuación.

Becker (1964), tuvo como uno de sus objetivos estudiar el capital humano, considerado el conjunto de capacidades productivas que un individuo adquiere por acumulación de conocimiento general y específicos. 
Entre los principales conceptos se hallan los gastos en que incurren los individuos en educación el entrenamiento en el trabajo, el cuidado de la salud y la migración. Concluye que las ganancias económicas presentan una correlación positiva con la educación y el entrenamiento. Por lo tanto el gasto educativo debe ser eficientemente utilizado.

El estudio de Mincer (1974), tuvo como objetivo hacer un análisis de los efectos sobre la los ingresos de la educación. Mincer precisó la importancia del ingreso-edad e ingreso-educación, llamada funciones de ingresos mancerinas a los que se añade la experiencia en el mejoramiento de los ingresos. El modelo de Mincer econométricamente puede presentarse como una ecuación en donde se estudia cómo afectan al ingreso del individuo dos variables explicativas los años de escolaridad y la experiencia laboral. En este modelo se observa que "cada año adicional" que el individuo invierta en educación le genera ingreso mayor al integrarse al mercado laboral y como participa la variable experiencia.

El estudio de Beyer (1999), tuvo como objetivo identificar las variable más significativas que afectan al coeficiente de Gini en Chile. Para ello ensaya una ecuación de mínimo cuadrados ordinarios del tipo:

$$
\begin{aligned}
\text { Gini, } t=\beta 0, t & +\beta 2 y i, t+\beta 2 y 2 i, t+\beta 3 E P i, t+\beta 4 \text { ES i, t }+\beta 5 \text { EU i, t } \\
& +\beta 6 \text { RETU } i+M R \lambda+M R \delta+\mu i,
\end{aligned}
$$

Dónde: Gini, t es el coeficiente de Gini, yi, t es el logaritmo natural del Producto Bruto per cápita. EPi,t, ESi,t y EUi,t corresponden a los "años promedios de educación primaria, secundaria y superior" de la población mayor de 15 años respectivamente.Dicho modelo ha servido como uno de los que se han utilizado de referencia para el modelo de la presente investigación. Beyer concluye que "Si Chile no es capaz de elevar sustancialmente la calidad de su educación no se avanzará en la reducción de las desigualdades. Frente a esta realidad los efectos de otras políticas, si existen, son apenas marginales. Los altos retornos relativos de la educación universitaria en Chile son, en una proporción importante, el resultado de deficiencias de nuestro sistema educacional que se hacen cada vez más notorias “(p. 129). Beyer señala además que mientras en Europa una persona con educación universitaria gana 1,8 veces lo que gana una persona solo con estudios de básicos, en Chile esa relación es de 5,5. 
Uno de los aportes más importantes de Amartya Sen es el concepto del enfoque de las «capacidades», se refiere a las capacidades de las que cada persona dispondría para poder convertir sus derechos en libertades reales. Una de las condiciones vitales es el acceso a la educación. Un gobierno tiene que ser juzgado en función de las capacidades concretas de sus ciudadanos. La eficiencia en el gasto del Estado es de suma importancia y se puede medir mediante la relación de años de estudios concluidos y su relación con la reducción de desigualdades.

Luego de los aportes básicos citados de Becker, Mincer, Beyer, y Sen, han surgido muchos estudios sobre la importancia de la educación en el crecimiento económico, por lo tanto en la reducción de las desigualdades. Uno de ellos es interesante para los fines del estudio es el de Villalobos y Pedroza (2009) quienes abordan un recorrido de la teoría del capital humano desde sus inicios en la posguerra hasta estudios recientes, centrando el análisis en el papel de la educación en la formación. Entre las conclusiones de ese estudio para los fines del presente documento interesa señalar que: "existen diferentes modelos y métodos para medir la utilidad de la inversión en capital humano, representan diferentes caminos para llegar al mismo fin, que se traduce en el análisis costo beneficio, que es la herramienta que ha empleado la teoría del capital humano para calcular las tasas de retorno de la inversión en educación. Otro punto se refiere a que los hacedores de política pública comparan costos contra beneficios con el fin de destinar recursos solo a actividades con mayor rentabilidad, lo cual es debatible, porque estas decisiones se fundamentan en juicios de valor sin considerar otros factores que representan beneficios no pecuniarios que es difícil medir de manera cuantitativa, por lo que, sobre todo los opositores de este enfoque, consideran este aspecto como su mayor debilidad" (p. 301).

El estudio de Torres Zambrano (2013), tuvo como objetivo realizar una estimación de las tasas de retorno de educación en el Perú según los niveles educativos para el año 2013, con énfasis en la educación superior universitaria y no universitaria. Este estudio concluye que "Una persona con mayor nivel educativo presenta una mayor tasa de retorno a la educación producto de su inversión reflejada en un mayor nivel de ingreso. La menor tasa de retorno del nivel superior universitario y no universitario entre los años 2004 y 2013, se puede explicar desde el mercado laboral, la 
oferta de empleo para las personas con carreras universitarias no crece al mismo ritmo de las personas egresadas. Asimismo, otro factor serio desde el sistema educativo es que no responde a la demanda de los sectores productivos, y por tanto no se ve un efecto positivo de la educación en el ingreso, en algunos sectores económicos" (p.80).

El estudio de Valdivia (2007) tuvo como objetivo analizar las posibles desigualdades derivadas de la relación entre pobreza y rendimiento académico de los distritos de la Provincia de Chacabuco y como hipótesis planteaba que los distritos pobres y segregados no tienen una relación directa con aquellos de bajo rendimiento en educación "...ha quedado claro como en general los establecimientos educacionales con bajos logros se localizan indistintamente en áreas con y sin problemas socioeconómicos...” (p.254), “...teniendo en cuenta las situaciones de pobreza y los bajos rendimientos en educación, la elaboración de estrategias integrales no solo deben tener en cuenta los factores determinantes, sino que además considerar junto a éstos, como en el caso de la solución de la pobreza, factores relacionados con otras áreas sociales, inversión en el territorio y el área económica-productiva, a fin de que éstas sean lo más cercanas posibles a los planteamientos de la teoría del desarrollo sustentable" (p. 255).

El estudio de Bolonotto (2007), tuvo como objetivo responder a la pregunta de si en México se verifica el denominado sheepskin effect o premio a la obtención del título. La hipótesis pretendió analizar el efecto del premio por la obtención del título en los ingresos. Concluye su estudio señalando que "Los resultados encontrados son consistentes con las predicciones sobre mayor presencia de los sheepskin effects en los sectores de administración pública, salud y educación debido a que en estos el título sirve como requisito de admisión para ciertas ocupaciones (p. 36).

El estudio de Ventura Neyra (2012), tuvo entre sus objetivos específicos: encontrar qué funciones del gasto público se encuentran más relacionadas con la heterogeneidad en los retornos (reducción de desigualdad de los ingresos), sus hipótesis específicas se refieren a que existen diferencias considerables en los retornos a la educación en las regiones del Perú y que el efecto se da a través de las funciones del gasto público que afectan los niveles de capital humano en las regiones. El estudio concluye la existencia de heterogeneidad en los retornos (reducción de la desigualdad de los 
ingresos) de la educación a nivel regional y cómo estas se ven reforzadas por el nivel de gasto público ejecutado en ellas. El plus del presente estudio respecto a Ventura Neyra es la incorporación de variables como tasa de estudios concluidos de educación primaria; tasa de analfabetismo, además el presente estudio no usa variables dummies.

El estudio de Freire y Teijeiro (2010) tuvo como objetivo mostrar los rendimientos de la educación de la población en los jóvenes de 16 y 34 años, de las zonas geográficas de Ferrolterra, Bergantiños y Costa da Morte, de la Comunidad Autónoma de Galicia. Este estudio concluye que " se evidencia la relación existente entre la escolaridad y los ingresos laborales apoyándonos en las ecuaciones de ingresos de Mincer y que la relación entre rendimientos y escolarización (medida como años de estudio completados) nos muestra un rendimiento de la educación del 5,36\%, un poco más bajo que los resultados de las investigaciones revisadas.........cuando analizamos el efecto de otras variables potencialmente explicativas, el rendimiento de la educación baja hasta un 4,49\%, lo que nos indica que existen otras variables que afectan de manera significativa a los salarios" (p.300). A diferencia de nuestro estudio Ferroltera realiza una encuesta y utiliza 15 variables dummy además de la variable años de experiencia laboral.

El objetivo del estudio de Paz, Paredes y Quilla (2016) fue medir econométricamente los retornos de la educación (analizan en general solo el número de años de estudio de cada persona) de los jefes de hogar de la región de Puno, en base a la Encuesta Nacional de Hogares 2011 - 2015. Los autores concluyen señalando que: "La inversión en educación en la región de Puno es muy rentable, porque aumenta la productividad de jefes de hogar y conlleva a la obtención de un flujo de ingresos netos positivos y considerables en magnitud" (p. 455).

En el estudio de Vargas y García (2017), cuyo objetivo fue analizar y determinar la incidencia del capital humano en los procesos de descentralización de la educación en el municipio de Tlajomulco de Zúñiga, Jalisco, se halla que el ingreso aumenta en promedio $5.2 \%$, y que la experiencia incrementa en $5.6 \%$ el ingreso de los individuos anualmente. La variable experiencia se construyó con base al tiempo que el individuo ha dedicado a trabajar en el mercado y se calculó restando de la edad los grados de escolaridad. 


\subsection{Análisis de los dispositivos legales y opiniones de expertos en educación.}

Con la finalidad de que el estudio no sea solo cuantitativo y se sostenga en lo que sucedió en la realidad se consideró conveniente acompañarlo de un análisis de los dispositivos legales y opiniones de expertos en educación.

De las revisiones de las principales políticas educativas que pretenden entre sus objetivos coadyuvar a la disminución de la desigualdad de los ingresos a través de una mejor oferta de servicios educativos del estado podemos mencionar las siguientes:

Ley № 27558, Ley de Fomento de la Educación de las Niñas y Adolescentes Rurales (año 2001): establece disposiciones para la promoción de la equidad en el acceso y calidad del servicio educativo en la educación inicial, primaria y secundaria para niñas y adolescentes rurales y define los objetivos y estrategias de la educación rural; la capacitación de los profesores rurales; la participación de las familias; y el presupuesto.

Ley 28044, Ley General de Educación (año 2003): se refiere a las responsabilidades de la sociedad educadora para la formación integral de las personas, bajo un enfoque intercultural, descentralizado, intersectorial y mejora continua permanente.

Ley 28983: Ley de Igualdad de Oportunidades entre Mujeres y Hombres (año 2007): impide la discriminación en todas las esferas de la vida, pública y privada, apoya las libertades fundamentales en las esferas política, económica, social, cultural.

Ley 30220, Ley Universitaria (año 2014): promueve el mejoramiento continuo de la calidad educativa de las universidades como entes fundamentales del desarrollo nacional.

Ley 30512, Ley de Institutos y Escuelas Superior de Educación (año 2016): considera que la formación de calidad para el desarrollo integral de las personas responda a las necesidades: del país, del mercado laboral y del sistema educativo y, su articulación con los sectores productivos.

Decreto Supremo № 012 -2020- MINEDU, Política Nacional de Educación Superior y Técnico Productiva (año 2020): define la apuesta del 
Estado al 2030 para ampliar el acceso y mejorar la calidad de la educación superior, en conjunto con lo técnico-productivo.

Se aprecia importantes modificaciones legales de política educativa a lo largo del periodo de estudio. A partir del año 2001 puede inferirse que se inició un "periodo de reformas" o mejoras en la política educativa del Perú en el presente siglo. Se aprecia en el periodo de estudio: 2007-2017 se han ido implementando un conjunto de políticas dirigidas a integrar entre ellas diferentes elementos que una política única conjunta y sistémica debe incorporar "desde un principio". Se infiere de ellas que el Perú ha vivido periodos de políticas imprecisas, incompletas y no integradas, y que la emisión de los dispositivos legales del periodo en su conjunto pretende una educación moderna, con una formación integral, especializada, intercultural, inclusiva y de igualdad.

\subsection{Análisis de Opiniones de Expertos.}

En paralelo a la emisión de los diversos dispositivos legales en el sector educación,las opiniones de expertos en el tema educativo pueden resumirse de la siguiente manera: Los expertos manifestaban que las estructuras de ingreso de los docentes no recibían los mismos retornos de calificaciones que los del resto del mercado laboral (Saavedra,2004); que el sistema educativo estaba en crisis (Vexler, 2004); en el año 2008 se relaciona que la inequidad en educación tienen una enorme relación con el modelo de desarrollo y la situación actual en la que se encuentra cada región (Aguirre, 2008) y, que se requiere educación que facilite las competencias para adaptarse a los cambios laborales y para la autogeneración de empleo (Fernández,2008). Además luego de la implementación de las primeras mejoras en la política educativa los expertos manifiestan que es imposible lograr la inclusión social si se mantiene el nivel educativo que tenemos (Parodi, 2013). Asimismo los jóvenes perciben que si van a un instituto conseguirán trabajo más rápido (Lescano, 2014); que se sigue reconociendo la mirada etnoceentrica del Estado, se percibe que la educación rural no ha sido una prioridad y que no se conoce la problemática rural en todas sus dimensiones (Ríos, 2016). Asimismo hay estudios que evidencian que pobres o pobres extremos muestran en general menores niveles de acceso o logro educativo (Guadalupe, 2017); otros estudios muestran que continúa la inequidad en el acceso a servicios educativos por cuanto el Perú 
no tiene un plan nacional de infraestructura intersectorial, el déficit de infraestructura educativa es de S/100 mil millones cinco veces más de lo que se ha invertido en gasto de capital en educación en los últimos cinco años $y$, que se requiere invertir en las llamadas habilidades del siglo XXI - habilidades socioemocionales, digitales y en las áreas de ciencias, matemáticas, ingenierías y artes -cada vez más necesario para lograr formar los ciudadanos que necesitamos en el siglo XXI (Vegas, 2019).

\section{Métodología.}

El presente estudio es no experimental, desarrolla un diseño longitudinal y transversal, debido a que busca analizar cambios a través del tiempo con determinadas variables o en las relaciones de estas. Se utilizan datos del periodo 2007 al 2017 para hacer el análisis, tomando como información estadística del Instituto Nacional de Estadística (INEI) y del Ministerio de Educación (Minen). Se utilizó el aplicativo Stata.

\section{Modelo econométrico seleccionado. -}

A partir del estudios de Beyer (1999), y complementándose, con las investigaciones que se mencionan en la revisión de literatura, principalmente las de Torres Zambrano (2013), Ventura Neyra (2012), Freire y Teijeiro (2010) se seleccionaron las variables de estudio considerando la disponibilidad de información. A partir de ello se consideró conveniente estructurar el siguiente modelo:

$$
\operatorname{Gini}_{i, t}=\beta_{0, t}+\beta_{1} y_{i, t}+\beta_{2} E P_{i, t}+\beta_{3} E U_{i, t}+\beta_{4} P E A_{i}+\beta_{5} T A+u_{i, t}
$$

En donde las variables de modelo son: Gini $i_{i, t}$ nivel de desigualdad de los ingresos; $y_{i, t}$ : logaritmo natural del salario por hora; $E P_{i, t}, E U_{i, t}$ : corresponden a la tasa de alumnos que concluyeron el nivel de educación primaria y superior, respectivamente; PEA: población económicamente activa; TA: tasa de analfabetismo.

Entre las pruebas consideradas para el tratamiento de la información podemos señalar las siguientes: el test de Hausman, test chi cuadrado que determina si las diferencias son sistemáticas y significativas entre dos estimaciones, el test de Heterocedasticidad, $y$ el test de Autocorrelación con el método de Wooldridge. 


\section{Resultados}

Se superaron los parámetros de todos los test estadísticos y econométricos para evitar una medición espurea y para que esta sea representativa de la realidad.

La hipótesis de trabajo consistió en afirmar que las políticas educativas tuvieron un efecto o impacto bajo en la reducción de las desigualdades de los ingresos en los años 2007 al 2017. Se concluye que la hipótesis se cumple.

\section{Tabla № 1}

Resultados Econométricos

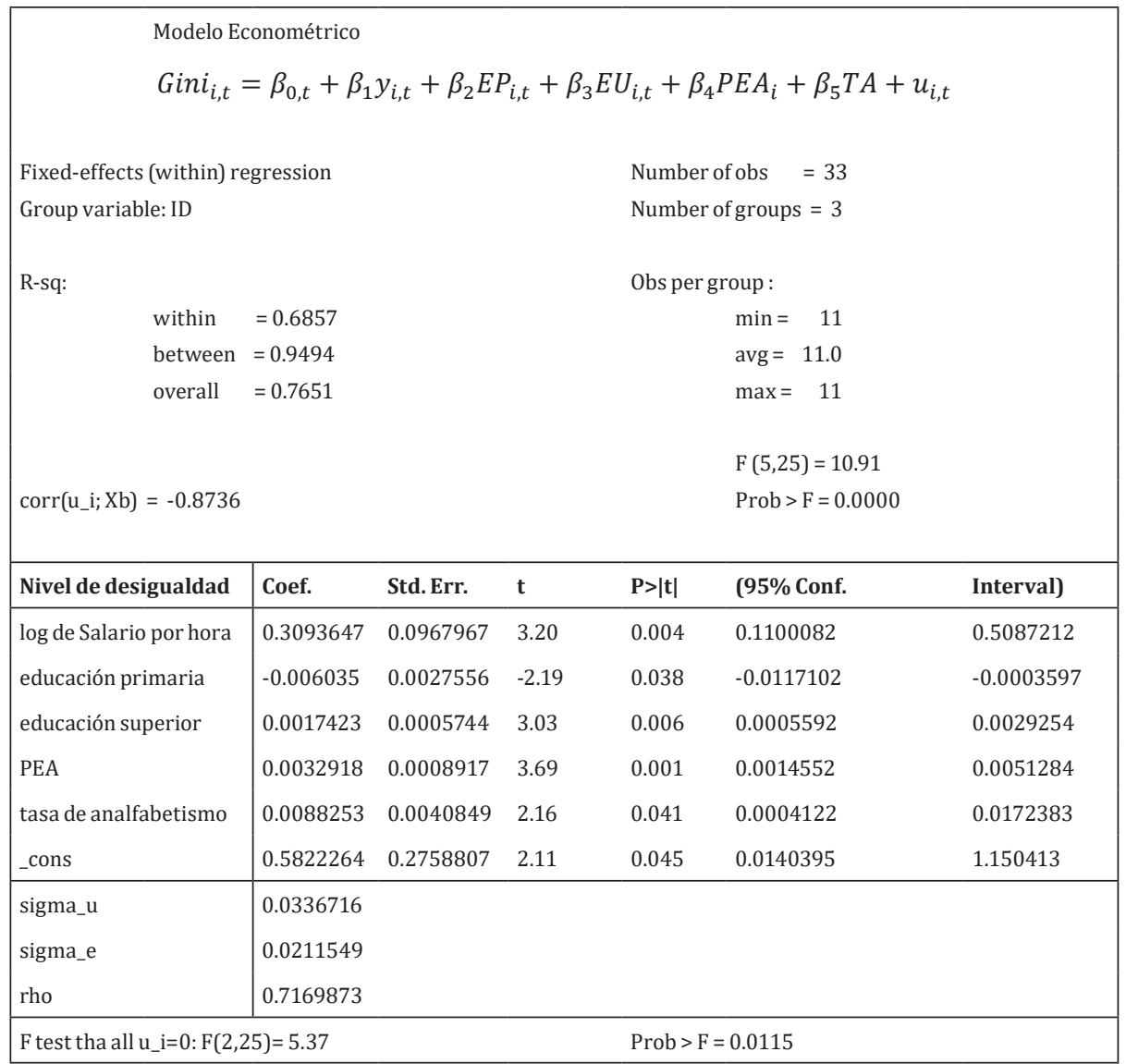


Los resultados de la prueba $\mathrm{F}$ indican que el conjunto de variables es representativo. Todas las variables presentan una prueba $\mathrm{p}$ - valor menor a 0.05, estadísticamente son significativas. Los coeficientes para el estudio de la incidencia de las políticas educativas: educación primaria y superior y, analfabetismo son pequeños, a excepción de la variable salarios por hora. El crecimiento de la población económicamente activa muestra un coeficiente también bajo.

La figura № 2 y la tabla № 1, los salarios por hora crecen sostenidamente y es la variable que alcanza un mayor coeficiente de impacto (0.3093647). En tal sentido, ha sido comparativamente la política salarial más eficiente en la reducción de las desigualdades en la regiones en estudio respecto al resto de las variables seleccionadas de política educativa, lo cual está asociado a lo que encontró Torres (2013), quien señala que el sistema educativo no responde a la demanda de los sectores productivos.

En relación a las variables que afectan directamente la política del gasto educativo si se observa la figura $\mathrm{N}^{\circ} 3$ el comportamiento de la variable alumnos que concluyeron en educación primaria tiene una tendencia creciente lo que evidencia que las administraciones de gobierno regionales se han preocupado en su atención, pero dicha mejora no ha sido sostenida en las tres regiones. En la evidencia empírica del modelo (véase tabla №1) el signo es negativo y es bajo el coeficiente encontrado (0.006035) para la variable educación primaria. Dicha variable no incide fuertemente, se refiere a los primeros años de formación y, por cuanto su efecto para mejorar el nivel de calificación de los trabajadores tarda en manifestarse. Esto no sucede en países avanzados en donde la oferta educativa está más articulada con los sectores productivos.

Según se observa en la figura $\mathrm{N}^{\circ} 4$ el nivel de educación superior concluida a partir del año 2008 tiene una evolución irregular para las tres regiones del estudio. En la contrastación econométrica el signo de la variable educación superior es positivo, lo que se relaciona directamente a lo encontrado por Torres (2013). Sin embargo, en el caso peruano (véase tabla №1) el coeficiente es bajo (0.0017423), de lo que difiere de lo encontrado por Freire y Teijeiro (2010) en donde la relación entre rendimientos y escolarización muestra un rendimiento de la educación del 5,36\% superior. Por ello es recomendable establecer medidas de incentivos de la política económica 
- educativa a los que se refiere Bolonotto (2007): la mayor presencia de los sheepskin effects (premio a la obtención del título).

La tasa de analfabetismo conduce a reflexionar de que si bien la tasa se ha ido reduciendo en el periodo de estudio (véase Figura $\mathrm{N}^{\circ} 5$ ) su bajo coeficiente (0.0032910) manifiesta un bajo efecto en la reducción de la desigualdad de los ingresos (véase tabla №1).

Finalmente obsérvese en la figura $N^{\circ} 6$ que la población económicamente activa, por su bajo coeficiente $(0.0088253)$ en el cálculo econométrico incide muy poco en la desigualdad de los ingresos.

\section{Discusión}

Los resultados encontrados evidencian que durante el periodo de estudio se dieron reformas o mejoras en la política educativa y del propio gasto educativo del Perú en el presente siglo, y que las opiniones de los expertos presentadas son válidas.

Las opiniones de los expertos es importante por cuanto tienen especialización experiencia en la materia, han desempeñado cargos públicos nacionales o cargos en instituciones internacionales de primer nivel, cátedras, investigación,periodismo especializado etc. Sus apreciaciones son importantes por cuanto son veedores o testigos presenciales de lo que aconteció en el periodo de estudio.

Las opiniones de los expertos revisados corroboran los resultados econométricos encontrados: Vexler (2004) se refirió a que el sistema educativo se encontraba en crisis y que los salarios de los profesores eran bajos e injustos, Saavedra (2004) preciso sobre las diferentes estructuras de salarios (injustas y que requieren cambios) en los profesores, Parodi (2013) recomendó un cambio drástico en la educación, Lescano (2014) preciso la importancia de las carreras técnicas para conseguir un trabajo más rápido para reducir la desigualdad de los ingresos y que la educación rural no ha sido una prioridad, que no se conoce la problemática rural en todas sus dimensiones, Rios (2016) se refirió a modelos estandarizados que no correspondían a las necesidades de las regiones, Aguirre (2008) y Villegas (2019) se refirieron a la inequidad en el acceso a los servicios educativos, Vegas (2019) a que se debe invertir en educación a lo largo de todo 
el ciclo de vida y que se debe invertir en las llamadas habilidades del siglo XXI:habilidades socioemocionales, digitales y en las áreas de ciencias, matemáticas, ingenierías y artes. Las diferentes opiniones de expertos están relacionadas a reducir las desigualdades de los ingresos.

La implementación de las políticas está en camino, se encuentra en su fase de iniciación en el presente siglo, la educación debemos concebirla como un producto de largo aliento, un peruano estará completamente preparado luego de culminar satisfactoriamente su educación en los siguientes niveles: 10 años en el nivel escolar, 3 años en el nivel de institutos superiores y 5 años en universidades, 2 y 3 años en los niveles de maestría y doctoral. Lo que debe hacerse es articular la fuerza laborar objetivo potencial por regiones con los niveles de desarrollo progresivos deseados considerando el nivel educativo que se requiera para ello y priorizando los presupuestos y políticas en función de las necesidades de cada región. Es importante destacar que se requiere también formar a profesionales y técnicos en institutos.

Finalmente, en el presente estudio se ha podido comprobar la importancia de los aportes de Becker (1964) Mincer (1974), Beyer (1999) en los cuales se destacan la importancia de la educación y sus variables de estudio en el desarrollo económico, aun cuando el resultado de los efectos encontrados en el presente estudio es bajo. Asimismo la comparación de los resultados del presente estudio respecto a las investigaciones de: Torres Zambrano (2018), corroboran los resultados del mencionado autor en el sentido de que la educación logra tener una mayor participación en el crecimiento de la economía; de Ventura Neyra (2012), se corroboro que el gasto público en educación tiene un efecto en el retorno de la educación medido mediante la reducción del coeficiente de Gini; de Freire Seoane (2010), se corroboro que los años de educación si tienen un efecto en el ingreso de las personas.

\section{Conclusiones y Recomendaciones}

\section{Conclusiones}

En general se puede apreciar que las mejoras en las políticas del gasto en educación han tenido un bajo efecto o impacto, durante el periodo de 
estudio en la reducción de la desigualdad de los ingresos para las regiones estudiadas. Asimismo después de haber vivido largos periodos de políticas educativas imprecisas, incompletas y no integradas, nos encontramos en un "periodo de inicio" de las reformas educativas, y que su verdadero efecto se podrá apreciar en un periodo mayor de análisis, siempre que por parte del Estado, exista un mayor control y seguimiento de los objetivos y metas de ellas, tanto en el aspecto cuantitativo como cualitativo.

Ha sido útil el uso de las variables del modelo: el nivel de desigualdad de los ingresos, el salario por hora, el nivel de educación primaria concluida, nivel de educación superior concluida, nivel de población económicamente activa, tasa de analfabetismo.

Que el bajo efecto o impacto de la política educativa en la mejora de ingresos laborales y en una distribución menos desigual, se mantendrá si el Estado no considera las opiniones de expertos en el diseño de las políticas, y realiza un seguimiento a ellas.

En adición al modelo planteado que tuvo un enfoque principalmente de variables de política educativa que influyen en el acceso educativo, se deben desarrollar otros modelos que evalúen la mejora cualitativa de la provisión de servicios educativos, principalmente el de competencia y habilidades.

\section{Recomendaciones}

El Estado debe continuar con un mayor control y seguimiento a la implementación de las políticas educativas mencionadas en el presente documento y otras políticas que sean de especial interés por parte de los agentes involucrados entre ellos: alumnos, padres de familia, educadores, funcionarios públicos de las instituciones del Estado tanto a nivel nacional como regional y distrital.

Revisar permanentemente si lo programas educacionales mantiene en su aplicación el enfoque de una educación moderna, con la inclusión de las llamadas habilidades del siglo XXI, con una formación integral, especializada, intercultural, inclusiva y de igualdad, que propicie la reducción de las desigualdades de los ingresos. 
Actualizar de manera permanente al profesorado, considerándolo como principal agente del mejoramiento educativo.

Articular los programas educativos dentro de las regiones mediante la participación de todos los actores involucrados: padres de familia, funcionarios públicos, autoridades distritales, regionales y nacionales y principalmente el sector empresarial público y privado.

Revisar la literatura del presente artículo por cuanto servirá para una mejor ilustración y análisis crítico de las políticas y estrategias educativas.

Nota.- Los autores manifiestan que no existe conflicto de interés, por apoyo financiero o material por parte de alguna institución estatal o privada.

\section{Referencias Bibliográficas}

Aguirre, Noel y otros, (2008), Una mejor Educación para una mejor sociedad: Inequidad y educación en América Latina, Fe y Alegría, p. 35, recuperado de: https://www.feyalegria.it/sites/default/files/UnaMejorEducacion.pd

Becker Gary. (1964) El Capital Humano - Un Análisis Teórico y Empírico referido fundamentalmente a la educación. https://papers.ssrn.com/sol3/papers. cfm?abstract_id=1496221

Beyer, Harald (1999), educación y desigualdad de ingresos: una nueva mirada, Serie Documentos de Trabajo, Centro de Estudios Públicos, N²97, Chile, agosto 1999, http://www.educandojuntos.cl/wp-content/uploads/2015/11/ educacion-y-desigualdad-de-ingresos-una-nueva-mirada-harald-beyercep-2000.pdf

Bolonotto Fernández, Lise (2007), Las Tasas de Retorno a la Educación: El Caso Mexicano, Pontificia Universidad Católica de Chile -Instituto de Economía, Diciembre 2007, http://economia.uc.cl/docs/tesis_lbolonotto.pdf

Guadalupe, León, Rodríguez y Vargas (2017), Estado de la educación en el Perú, FORGE, recuperado de: http://www.grade.org.pe/forge/descargas/Estado\%20de\%20la\%20educaci\%C3\%B3n\%20en\%20el\%20Per\%C3\%BA.pdf

Fernández Ludeña, Alejandro y otros, (2008), Una mejor Educación para una mejor sociedad: La educación para el trabajo en un mundo globalizado. Una visión desde Fe y Alegría, p.106, en UNA MEJOR PARA UNA MEJOR EDUCACIÓN SOCIEDAD Propuestas para el diálogo y la transformación educativa en América Latina y el Caribe, recuperado de: https://www.feyalegria.it/ sites/default/files/UnaMejorEducacion.pdf 
Freire Seoane María Jesus y Teijeiro Álvarez, Mercedes. (2010). Las ecuaciones de Mincer y las tasas de rendimiento de la educación en Galicia. Chapter 14 in Investigaciones de Economía de la Educación, 2010, vol. 5, pp 285-304 from Asociación de Economía de la Educación, Universidad de la Coruña, España. http://repec.economicsofeducation.com/2010zaragoza/05-14.pdf

INSTITUTO NACIONAL DE ESTADISTICA (INEI), http://escale.minedu.gob.pe/ indicadores; http://iinei.inei.gob.pe/microdatos/Lescano, Luis (2014) Jóvenes están colocando a los institutos técnicos a la par de cualquier universidad, SINEACE, Perú, recuperado de: https://www.sineace.gob.pe/ jovenes-estan-colocando-a-los-institutos-tecnicos-a-la-par-de-cualquieruniversidad/

Mincer Jacob. (1974). Escolaridad, experiencia y Ganancias. Oficina Nacional de Investigación, Nueva York. https://econpapers.repec.org/bookchap/nbrnberbk/minc74-1.htm

MINISTERIO DE EDUCACIÓN; Estadística Escale; http://escale.minedu.gob.pe/ indicadores.

Ñopo (2018), ¿Aumentó la desigualdad en el Perú? https://elcomercio.pe/econo$\mathrm{mia} /$ peru/aumento-desigualdad-peru-noticia-514678-noticia/?ref=ecr

OCDE, (2016). Avanzando hacia una mejor educación para Perú. Organización para la Cooperación y el Desarrollo Económicos, Lima.

Parodi, Carlos (2013), Calidad de la Educación en el Perú, Perú. http://blogs.gestion.pe/economiaparatodos/2013/12/calidad-de-la-educacion-en-el.html

Paz,Rene; Paredes Mamani, Quilla Manrique Juan Carlos (2016), Retornos a la educación de los Jefes de Hogar en la Región de Puno, 2011 - 2015, RIA: Revista de Investigación Altoandina, 2016; Vol 18 Nro 4, http://www.scielo. org.pe/pdf/ria/v18n4/a08v18n4.pdf

PCM: Ley № 27558, Ley de Fomento de la Educación de las Niñas y Adolescentes Rurales (año 2001); Ley 28044, Ley General de Educación (año 2003)

Ley 28983: Ley de Igualdad de Oportunidades entre Mujeres y Hombres (año 2007)

Ley 30220, Ley Universitaria (año 2014); Ley 30512, Ley de Institutos y Escuelas Superior de Educación (año 2016); Decreto Supremo № 012 -2020- MINEDU, Política Nacional de Educación Superior y Técnico Productiva (año 2020);http://www4.congreso.gob.pe/historico/cip/materiales/profesorado 
Ríos, Chris, (2016), CADE por la educación: La educación rural rumbo al 2021, en IPAE-CADE por la educación, La educación fuera de la caja, p. 14, recuperado de: https://www.ipae.pe/wp-content/uploads/2017/05/revista_cade_05-09.pdf

Saavedra, Jaime y otros, (2004), ¿Es posible Mejorar la educación? Evidencias y posibilidades, GRADE, p. 231, recuperado de: https://www.grade.org.pe/wpcontent/uploads/LIBROGRADE_ESPOSIBLEMEJORAREDUCACION.pdf

Torres Zambrano, Manuel (2013), Relación entre educación e ingreso: los retornos a la educación en el perú estimados para el año 2013 Trabajo de investigación para optar el Título Profesional de Economista, https://repositorio. ulima.edu.pe/handle/20.500.12724/7134

Valdivia Beltrán, Paulina Ingrid (2007), La pobreza y su relación con los bajos rendimientos en educación: un ejemplo de desigualdad a nivel distrital, provincia de Chacabuco, comunas de colina, lampa y til, Memoria para optar al Título Profesional de Geógrafa,Universidad de Chile Facultad de Arquitectura y Urbanismo Departamento de Geografía Chile, Enero del 2007, http:// repositorio.uchile.cl/bitstream/handle/2250/101084/027_valdivia_p. pdf? sequence $=3 \&$ isAllowed $=y$

Vargas-Hernández, José G. y, García Oliva, Víctor Hugo (2017),Capital humano y descentralización de la educación. El caso para Tlajomulco de Zuñiga, Jalisco, Ens. Econ. vol.27 no.50 Medellín Jan./June 2017,Universidad de Guadalajara, Guadalajara, México. http://www.scielo.org.co/scielo.php?script=sci_ arttext\&pid=S2619-65732017000100181\&lng=en\&nrm=iso\&tlng=en

Vegas, Emiliana. (Febrero 2019). Mejor Inversión para Mejores Resultados Educativos. 18-10-19, BID Sitio web: https://blogs.iadb.org/educacion/es/mejorinversionmejoresresultados/

Villegas, María Cecilia (2019). ¿Cómo les explicamos a los padres que deben mandar a sus hijos a colegios que no son seguros para ellos? Colegios que ni siquiera cuentan con los servicios básicos. 18/10/19, de Peru21 Sitio web: https://peru21.pe/opinion/educacion-398955-noticia/

Ventura Neyra, E. (2012). Análisis de la heterogeneidad en los retornos a la educación en función del gasto público regional. (Tesis de magister). Pontificia universidad católica del Perú. Tesis para optar el grado de Magíster en Economía, PUCP, http://tesis.pucp.edu.pe/repositorio/handle/20.500.12404/4894

Villalobos Monroy, Guadalupe y Pedroza Flores Reneé (2009), Perspectiva de la teoría del capital humano acerca de la relación entre educación y desarro- 
llo económico, Tiempo de educar, año 10, segunda época, número 20, juliodiciembre de 2009, Facultad de Ciencias de la Conducta de la Universidad Autónoma del Estado de México, Toluca, México, https://www.redalyc.org/ pdf/311/31112987002.pdf

Vexler,Idel (2004) Informe sobre la Educación Peruana Situación y perspectiva, OEI, https://studylib.es/doc/4855125/informe-sobre-la-educaci\%C3\%B3nperuana--\%E2\%80\%9Csituaci\%C3\%B3n-y-perspect

Unesco, (2015). Situación educativa en América latina y el Caribe: Hacia la educación de calidad para todos al 2015, Santiago de Chile.

Wikipedia, Amarthya Sen; https://es.wikipedia.org/wiki/Amartya_Sen

\section{Notas al final}

1 Artículo de Debate

2 Economista, Universidad de Lima, Lima, Perú. Master en Administración de Empresas y Doctor en Ciencias Económicas y Empresariales por la Universidad Autónoma de Madrid. Becas de Investigación en la Universidad Libre de Berlín - RFA y en la Universidad Luiggi Bocconi, Milán- Italia. Premio Nacional de Economía - (1983) Colegio de Economistas de Lima. Profesor Principal e Investigador responsable del Grupo de Investigación: Evidencia Empírica Jerarquización de la Facultad de Ciencias Económicas, Universidad Nacional Mayor de San Marcos, Lima, Perú. Calle Germán Amezaga 375, Lima, Perú. Email: rvillamonteb@unmsm.edu.pe

3 Economista de la Universidad Nacional Mayor de San Marcos Email: tomas.delgado@unmsm.edu.pe 
